\title{
SENTIDO, SINSENTIDO Y FILOSOFÍA EN WITTGENSTEIN
}

\author{
Anastasio ALEMÁN
}

Resumen: Uno de los tópicos más extendidos en torno a la filosofia de Wittgenstein (en especial la de su última fase) consiste en considerarlo meramente como un filosofo del lenguaje ordinario preso del dogma de que él lenguaje ordinario está bien como está' y que, como consecuencia de esto, reduce la tarea de la filosofia a tan solo describirlo sin intentar cambiarlo.

Mi proposito en estas páginas consiste en mostrar lo infundado y equivocado de esta difundida idea.

Abstract: One of the commonplaces about the philosophy of Wittgenstein (especially in the later period) consists of considering him merely as a philosopher of ordinary language unable to break away from the dogma 'ordinary language is in order as it is', and that consequently reduces the task of Philosophy to just the description of language with no attempt of reforming it.

In this paper I propose to prove how unfounded and mistaken this idea is.

Este artículo constituye, en su conjunto, una defensa de las siguientes tesis: (i) Wittgenstein a pesar de haber mantenido, tanto en el Tractatus (5.5563) como en los Cuadernos (p.57) y las Investigaciones (98), que el lenguaje ordinario está bien como está, es en realidad un reformador del propio lenguaje ordinario, y que lo fue a lo largo de toda su obra, a pesar de su explícita renuncia a este tipo de tarea (op. cit.,132).

(ii) Que lo reformó, al menos en su última fase, no proponiendo un modelo de lenguaje alternativo, sino clarificando el propio lenguaje ordinario y 
optando por ciertos usos ordinarios de términos cruciales que ocurren en él, por ejemplo 'verdadero' y 'falso', frente a otros usos que también ocurren en él pero que resultan incongruentes con los elegidos, v.g. con los tomados como paradigmáticos.

(iii) Que la opción por ciertos usos frente a otros está orientada por el legítimo objetivo que reconoce de «establecer un orden en nuestro conocimiento del lenguaje» (op. cit., 132), dado que una misma palabra puede desempeñar funciones lógico-conceptuales muy diferentes (op. cit., 11); [por ejemplo, la palabra 'es' en 'La rosa es roja' y en 'Dos más dos es cuatro' (op. cit., 558; 1969, p. 53)].

(iv) Que como consecuencia de esto W no se limitó meramente a describir el uso ordinario de los términos, en contra de sus repetidos pronunciamientos en sentido contrario (1988a, 124), sino que implícitamente recomendó ciertos usos al proscribir otros, tachándolos, explícitamente, de sinsentidos.

Conviene advertir de antemano que las cuatro partes en las que esta dividido este artículo no se corresponden unívocamente con cada una de las tesis anteriores. La defensa de esas tesis se encuentra diseminada, con diferentes matices, a lo largo de todo el artículo. No obstante, a fin de clarificar la estructura del texto en relación con las tesis mencionadas, debe señalarse lo siguiente: En la parte I se bosquejan brevemente las lineas principales de la concepción del mundo que, en mi opinión, subyacen a lo largo de toda la obra de Wittgenstein (aunque, de forma más explícita, en su fase madura) poniendola en conexión con una de sus distinciones fundamentales, a saber, la que hay entre expresiones con sentido y expresiones sin sentido. En la parte II analizamos detenidamente cómo trazó W esa distinción en el Tractatus, y los resultados alcanzados muestran que las tesis (i), (iii) resultan correctas respecto a su posición en esa obra. La parte III está dedicada a poner de manifiesto que $\mathrm{W}$ no mantuvo (en su fase madura) únicamente una concepción descriptivista de la filosofía, sino que cabe hablar razonablemente de que dejó lugar para una concepción que denominamos 'constructivista'. Esta distinción está en la base de la tesis (iv), cuya defensa aparece en la parte siguiente. Finalmente, la parte IV constituye una nueva defensa de las cuatro tesis principales del artículo, centrándonos, en este caso, en una de las nociones de W que, en lo fundamental, permaneció constante a lo largo de toda su obra: la noción de verdad. 
Una filosofía, o incluso, más generalmente, una concepción del mundo, entraña siempre una cierta clasificación última de las cosas.

A este hecho no se sustrae ni siquiera la filosofía de $\mathrm{W}$ aún a pesar de que pase comúnmente por constituir un caso paradigmático de filosofía asistemática. Sin duda este carácter asistemático hace más diff́cil ver las líneas de tal tipo de clasificación, pero dificultad no equivale, claro está, a imposibilidad.

La filosofía de W aparece, en principio, como una filosofía del lenguaje pero, como tal, encierra (pese quizás a sus intenciones) una cierta concepción del mundo. Una concepción que resulta bosquejada de un modo más bien negativo de expresarla: No existe un mundo independiente de entidades matemáticas o lógicas que la mente humana pueda descubrir de algún modo (usualmente mediante algún tipo de intuición), ni existe un mundo de valores objetivos que puedan percibirse mediante algún tipo de intuición moral o estética; ni existe, en general, un mundo de conceptos del que nuestras palabras y términos linguísticos no constituirían más que un pálido y tosco reflejo sonoro o gráfico.

No existe, en definitiva, un mundo de entidades abstractas y de relaciones entre ellas, independientes del lenguaje, que haría verdaderos, o falsos, a nuestros enunciados matemáticos, morales o, en general, conceptuales.

Pero todas estas ideas no son más que el resultado final de un largo y diffcil camino a través del análisis del lenguaje. No podía ser de otro modo. Una concepción del mundo, como cualquier teoría o interpretación, aparece expresada, necesariamente, en uno u otro lenguaje. Incluso el poeta más intimista es reconocido y apreciado por sus versos escritos o declamados, no por aquellos no expresados, si es que tiene algún sentido emplear aquí la expresión 'aquellos'.

Siendo esto así, la tarea de analizar el instrumento mediante el que se expresa cualquier concepción del mundo resulta inexcusable.

Pero puestos a abordar esa tarea parece claro que el primer problema consistirá en deslindar lo que forma parte del lenguaje de lo que no forma parte de él. En un principio parece claro que ciertas palabras - las que constituyen su vocabulario en cualquier momento dado- formarán parte de él y otras estarán excluidas como un hecho resultante de la practica lingüística de los 
usuarios de ese lenguaje. Pero el problema anterior aparece aquí de forma más concreta: ciertas combinaciones de palabras formarán oraciones bien formadas y con sentido en ese lenguaje, pero otro tipo de combinaciones quedarán excluidas del lenguaje como mal formadas o sinsentido. Por ejemplo, 'Leche me azúcar' (Wittgenstein), 'Estroncio bebe lenidad' (Russell), 'El sábado esta en la cama' (Ryle), 'Las verdes ideas duermen furiosamente' (Chomsky) ${ }^{1}$.

Quizás no haya lugar donde más clara resulte la prioridad de la distinción entre expresiones con sentido y expresiones sinsentido que en el caso de los denominados 'lenguajes artificiales', como por ejemplo el de la Lógica clásica. Efectivamente, antes de que podamos asignar un valor veritativo, o aplicar una regla de transformación (de inferencia), a una expresión dada, resulta preciso que esa expresión sea bien formada, esto es, que la particular combinación de signos que la constituye sea el resultado de la aplicación de las reglas de formación, reglas que determinan qué combinaciones de signos pertenecen al lenguaje en cuestión (v.g. tienen sentido en él) y cuales quedan excluidas como mal formadas, o sinsentido, en ese lenguaje. (A esta faceta del problema está dedicada la parte II).

La radicalidad de la distinción entre oraciones o, en general, expresiones con sentido y sinsentido, aparece claramente reconocida por $\mathrm{W}$ cuando indicó: "Tengo que comenzar con la distinción entre sentido y sinsentido. Nada es posible anterior a esto." (1969, p. 126-7), y su dificultad apreciada cuando al abordar el problema dijo, según nos cuenta Moore (1983, p.275), que tenia que zambullirse en algo terrible. No es de extrañar que percibiera la dificultad con toda su agudeza una vez abandonada la idea del Tractatus de que la lógica (la sintaxis lógica) determina los límites del discurso con sentido, y con ello el modo concreto en el que había trazado la distinción en esa obra. [Sobre el tránsito desde la noción de sintaxis lógica, concebida como determinante del discurso con sentido, a la más amplia noción de regla gramatical, que cumple similar función, puede verse Baker y Hacker (1992, 34-41)].

La importancia de la distinción no sólo ya había sido claramente reconocida por Russell con su teoría de los tipos logicos $^{2}$ sino que en cierto modo, y

1 Un análisis de las concepciones de RYLE, CHOMSKY, y otros, sobre los denominados, comúnmente, 'errores categoriales', se encuentra en mi (1985).

2 Esta teoría aparece como un intento de evitar las paradojas en la teoría de conjuntos mediante una precisa demarcación entre lo que tiene sentido decir y lo que no seria más que puro sinsentido. Por esto, la teoría de los tipos requiere una modificación de las propias reglas de for- 
como en tantas otras cuestiones, no es difícil encontrar trazas de la distinción en Aristóteles cuando distingue claramente entre lo que puede ser dicho y lo que no puede ser dicho de algo; por ejemplo, el término 'blanco', pero no su definición, podría decirse o predicarse de un cuerpo (Categorías, 2.2, 29-33). Sin embargo no nos vamos a ocupar aquí de los antecedentes históricos, sino que nos vamos a centrar preferentemente en la cuestión de cómo traza W la distinción entre uno y otro tipo de expresiones, en el papel que desempeña esta distinción en su forma de hacer filosofía, y de que modo la aplicación de la distinción, en casos concretos, acaba afectando a la idea de que el lenguaje ordinario está bien como está.

En el Tractatus esa idea se verá afectada como consecuencia de una rígida noción de sentido, junto con la idea de que es la sintaxis lógica la que determina qué combinaciones de signos cumplen con ese criterio de sentido. En su obra posterior, descartada esa rígda noción de sentido y abandonada su idea acerca del papel de la sintaxis lógica, continuará tachando de sinsentidos ciertas combinadiones de palabras; pero ahora ya no será en virtud de un criterio general de sentido, sino como resultado de analizar su uso en situaciones concretas, sean estas reales, o meramente posibles ${ }^{3}$. [La evolución del pensamien-

mación del lenguaje de la teorla de conjuntos, a diferencia, por ejemplo, de la solución menos radical de ZERMELO, que no requiere tal tipo de cambio, sino que se contenta con el mas sencillo de modificar el axioma 'ingenuo', e inconsistente, de comprehensión [ $\exists \mathrm{B} \forall \mathrm{x}\left(\mathrm{x} \in \mathrm{B} \leftrightarrow \Phi_{\mathrm{x}}\right)$ ] añadiendo la subfórmula ' $x \in A$ ' en el lado derecho del bicondicional, $y$ el cuantificador correspondiente al comienzo.Por supuesto que bajo estas aparentemente nimias diferencias, puramente sintacticas, subyacen concepciones substantivamente diferentes sobre la naturaleza de los conjuntos y sobre lo que tiene,o no,sentido decir sobre éllos.

Más detalles sobre este asunto pueden encontrarse en GARCIADIEGO (1992),y en IsHIGURO (1981) sobre la versión sintáctica de W de la teoría de los tipos.

3 G. HALLETT [1977], al igual que la mayoría de los comentadores de W, no aprecia que, pese a las explícitas declaraciones de W de que el lenguaje ordinario está bien como está (Tract., 5.5563; Invest., 98), en ambas obras W actua de modo contrario a esa idea, aunque por razones muy diferentes en cada una de ellas. Así para HaLLETT «El lenguaje ordinario, por consiguiente, está bien como está, no porque una maravillosa precisión y constancia subyazca tras su superficie [como según HaLLETT creyó el W del Tract.], sino porque tales cualidades 'ideales' son irrelevantes para los objetivos reales del habla." HaLLETT $(1977,160)$. Mi objección es que la razón que ofrece HALLETT no es suficiente para mantener la idea de que el lenguaje ordinario esta bien como esta; pues aunque es indudable que W abandonó el paradigma del lenguaje como cálculo sometido a reglas precisas, no obstante, hay otro modo de oponerse, en la practica, a esa idea, a saber, declarando sinsentidos ciertas combinaciones de palabras que los usuarios del lenguaje ordinario no calificarían así, por ejemplo, "Un círculo es una forma" (1969, p. 61) (Otros ejemplos de desviación del lenguaje ordinario se encuentran en las partes II y IV). 
to de W sobre esta distinción, así como su relación con la teoría de los tipos de Russell y con la teoría de Kant sobre los límites del conocimiento, se encuentra adecuadamente tratada en Glock (1996, 258-64)].

II

Así que nuestro problema inmediato es: ¿Cómo traza W la distinción entre expresiones con sentido y expresiones sinsentido?.

W usó en el Tractatus tres expresiones diferentes para calificar lo que hasta aquí hemos considerado dentro de la única categoría de las expresiones sinsentido, a saber, expresiones que 'no tienen sentido' (hat Keinen Sinn), expresiones 'carentes de sentido' (Sinnlos) y expresiones 'sinsentido' (Unsinnig).

¿Hay algún rasgo, o característica, que permita dar cuenta del empleo de uno u otro tipo de expresiones de otros, o se trata de una mera diferencia estilística?.

En el Tractatus hay una aguda diferencia entre aquellas expresiones a las que W califica de carentes de sentido ( Sinnlos) y las que considera como puros sinsentidos (Unsinnig): Tautologías y contradicciones son carentes de sentido (4.461), pero no sinsentidos (4.4611), mientras que las propias proposiciones del Tractatus son calificadas de sinsentidos (6.54). Sin duda aplicar tales calificativos a tales expresiones no puede por menos que producir cierta sorpresa. Uno no se extrañaría demasiado si se tachara de carente de sentido quizás algo como 'Clinton se rasca la oreja ahora o no se levantarán las sanciones contra Irak', pero sí algo como 'Clinton se rasca la oreja ahora o no es el caso que Clinton se rasca la oreja ahora'. Análogamente, cabría esperar que algo así como 'El sentido de una proposición es la raíz cuadrada del volumen que desplaza' fuera tachado de sinsentido, pero no la oración 'La proposición es una figura de la realidad; pues yo conozco el estado de cosas que representa si yo entiendo el sentido de la proposición' (4.021) ni a la oración ' 1 es un número', ni tampoco esperaríamos ver considerado como sinsentido la oración 'La mayor parte de las cuestiones y proposiciones de los filosofos proceden de que no comprendemos la lógica de nuestro lenguaje' (4.003), aún cuando tuviéramos algún reparo que ofrecer respecto a su verdad.

¿Cómo ha llegado W en el Tractatus a tan extraños resultados?.

En mi opinión los resultados anteriores a los que llega $\mathrm{W}$ constituyen una consecuencia inevitable de sus ideas acerca de cómo el sentido de cualquier 
proposición depende del sentido de las proposiciones elementales. Efectivamente, el sentido de una proposición elemental está constituido por el posible estado de cosas que representa, esto es, por la posible ordenación de objetos que representa. Así, si ese posible estado de cosas ocurre en el mundo, la proposición es verdadera, si no ocurre, es falsa. Por ello la negación de una proposición invierte el sentido de una proposición (5.2341); resulta verdadera en los estados de cosas en los que la proposición original resulta falsa. Puesto que el sentido de la proposición está constituido por un posible estado de cosas, no es necesario conocer su valor de verdad para poder entender su sentido (4.024). El sentido de la proposición se muestra ${ }^{4}$ en esta, pero no así su verdad (o falsedad), que depende de que las cosas estén en el mundo como la proposición afirma o dice que están (4.022).

$\mathrm{Si}$, por otra parte, la proposición es 'molecular', su sentido dependerá de su modo de composición mediante las conectivas lógicas y del sentido de sus proposiciones elementales componentes; así que, en definitiva, el sentido de una proposición molecular estará constituido por aquellos posibles estados de cosas (correspondientes a sus proposiciones elementales) en los que la proposición sería verdadera si ocurren efectivamente en el mundo real.

El punto crucial de todo este asunto es que, para W, el sentido de una proposición cualquiera consiste en lo que podríamos denominar sus 'posibilidades de verdad': Una proposición tiene sentido si puede (lógicamente) ser verdadera $y$ puede (lógicamente) ser falsa, esto es, si hay algún posible estado de cosas en el que resultaría verdadera si ocurriera y hay algún posible estado de cosas en el que la proposición resultaría falsa si ocurriera. [Resulta interesante reparar aquí que si en la frase anterior substituimos 'verdadera' por 'confirmada (parcialmente)', 'falsa' por 'refutada (parcialmente)' y 'estado de cosas' por 'experiencia', obtenemos la posición que mantuvo $\mathrm{W}$ posteriormente en las Bemerkungen (1964, p. 282s.)].

Considerado así el sentido de una proposición, resulta fácil entender lo que antes considerábamos extranio: ni las proposiciones tautológicas ni las proposiciones contradictorias (a las que califica de 'pseudo-proposiciones') tienen

4 Un detallado análisis de la distinción entre decir y mostrar, en el Tractatus, se encuentra en HIERRO (1992). 
sentido. Las primeras porque no pueden ser falsas ${ }^{5}$, las segundas porque no pueden ser verdaderas. No hay un posible estado de cosas tal que, si ocurriera, una tautología sería falsa (puesto que es verdadera, por definición, en todo posible estado de cosas); ni hay tampoco un posible estado de cosas tal que, si ocurriera, una contradicción resultaría verdadera, porque, como muestra su tabla de verdad, resulta falsa en todo posible estado de cosas correspondiente a sus propiedades elementales componentes. Por esto, ni tautologías ni contradicciones logran describir algo ['llueve o no llueve' no informa sobre ningún fenómeno meteorológico (4.461)], por esto carecen de sentido.

Sin embargo, tanto tautologías, como contradicciones, son el resultado de modos legítimos de composición de proposiciones elementales mediante las reglas del lenguaje simbólico, y en este respecto se diferencian claramente de expresiones como 'Sócrates es idéntico', a la que califica de sinsentido (unsinnig, 5.473), porque no hemos estipulado «ningrin significado a la palabra 'idéntico' como adjetivo" (5.4733), y por ello no forman parte del lenguaje. Uno se sentiría tentado a describir el asunto diciendo que tautologías y contradicciones son oraciones bien formadas de acuerdo con las reglas del lenguaje formal al que pertenecen, mientras que 'Sócrates es idéntico' no aparece como combinación bien formada de signos, y ésta sería la razón por la que W se cuida de agruparlas en categorías diferentes: expresiones carentes de sentido (Sinnlos) en el primer caso, y expresiones sinsentido (Unsinnig) en el segundo. También se incluiría en este segundo grupo la pseudo-proposición '1 es un número' y por la misma razón, aunque en este caso no resulte a primera vista tan patente; pues, para W, 'número', expresa un concepto formal, y el número 1 cae bajo ese concepto, pero "Que algo caiga bajo un concepto formal como su objeto, no se puede expresar por una proposición. Esto se muestra en el símbolo del objeto mismo. (El nombre muestra que él significa un objeto; el signo numérico, que él significa un número, etc.)» (4.126). El supuesto de este último párrafo es, simplemente, que, en el lenguaje ideal que W parece tener en mente, emplearíamos diferentes tipos de símbolos para diferentes tipos de entidades, y de este modo, reconociendo el tipo de símbolo, reconocemos lo que representa sin necesidad de decirlo explícitamente en el propio lenguaje. Así, siguiendo la convención usual de que los numerales ' 1 ', '2', etc., representan números, podemos prescindir de la oración ' 1 es un número',

5 En ACERo (1990) se analiza detenidamente este punto señalando lo que considera que son sus presupuestos metafísicos. 
como innecesaria (y por ello sin significado alguno según W, 3.328 y 5.47321), en el lenguaje lógico ideal. La situación resulta más clara si la comparamos con lo que ocurre con el lenguaje regimentado de la Lógica clásica: la expresión 'Px' es bien formada, pues se trata de una combinación de signos permitida por sus reglas de formación, y por ende forma parte de ese lenguaje; pero ' $\mathrm{x}$ ' es una variable de individuo' no es bien formada, no es una combinación de signos permitida por sus reglas de formación (aunque obviamente forma parte de la descripción metalingǘstica del propio lenguaje de la lógica; más precisamente, forma parte de las propias reglas de formación de expresiones bien formadas del lenguaje estándar de la Lógica de primer orden).

He hablado antes de 'tentado' al ofrecer la explicación precedente porque ese modo de presentarla plantea un problema inmediato, a saber, que W también incluye dentro de la categoría de las expresiones sinsentido a las propias proposiciones que componen el Tractatus:

Mis proposiciones esclarecen porque quien me entiende las reconoce al final como sinsentidos [unsinnig], cuando a través de ellas — sobre ellasha salido fuera de ellas. (Tiene, por así decirlo, que arrojar la escalera después de haber subido por ella).

Tiene que superar estas proposiciones; entonces ve correctamente el mundo. (6.54).

Así que resulta que las proposiciones que nos permiten nada menos que 'ver correctamente el mundo' son tan sinsentidos como 'Sócrates es idéntico', $y$, no obstante, logran comunicar pensamientos, cuya verdad le parece intocable y definitiva (Prólogo al Tractatus), desentendiéndose completamente de lo que dice en el resto del libro sobre las nociones de pensamiento y verdad. A todas luces esa parece ser una tarea excesiva para ser realizada por expresiones sinsentido.

Como acabamos de ver, el problema surge al incluir en la misma categoría, de las expresiones sinsentido, a expresiones como 'Sócrates es idéntico' y a las propias proposiciones (o pseudo-proposiciones) del Tractatus. ¿Cual puede haber sido la razón de W para proceder de esta extraña manera?.

En mi opinión, al proceder de ese modo, W está actuando de un modo radicalmente consecuente con lo que parece ser su idea de un lenguaje idealmente perfecto y con su noción sobre el sentido de una proposición. En un lenguaje idealmente perfecto (W habla de "Un lenguaje sígnico, pues, que obe- 
dezca a la gramática logica —a la sintaxis lógican(3.325)), sólo podrían construirse, o considerarse como combinaciones admisibles de signos, proposiciones bien formadas; o mejor dicho, las proposiciones bien formadas -las que formarían parte de ese lenguaje ideal- serían aquellas y sólo aquellas permitidas por sus reglas de formación. Primero habría que especificar cómo se forman las proposiciones elementales a partir de los signos simples, y a continuación cuáles son las reglas de composición de proposiciones elementales para construir proposiciones moleculares. Respecto a estas últimas (y prescindiendo del conocido problema de los cuantificadores) la situación parece clara, son las conectivas veritativo-funcionales, que pueden reducirse a una sola, las que establecen las conexiones entre las proposiciones elementales (5; 5.514; 5.515). Más problemáticas resultan las proposiciones elementales, porque éstas, nos dice, están formadas por concatenaciones de nombres simples (4.22), representados mediante las letras ' $x$ ', ' $y$ ' ' $z$ ' (4.24); pero no especifica el modo de conexión entre esos nombres, contentándose con indicar que la forma de la proposición elemental sería ' $\mathrm{fx}$ ', ' $\varphi$ (x,y)', etc. (4.24), y el problema aqui es que ni ' $f$ ', ni ' $\varphi$ ', representan nombres [menos aún nombres simples que denoten a un objeto simple; aunque eso no quiera decir que no denoten, (5.526)].Esto implica que ' $f x$ ', ' $\varphi(x, y)$ ', etc., no pueden representar la forma completamente analizada de una proposición elemental, que solo tendría que estar formada por nombres simples (representados por ' $\mathrm{x}$, ' $\mathrm{y}$ ', ' $\mathrm{z}$ ', etc.) 'en conexión inmediata' (4.221).

El punto fundamental es que W piensa que, en el análisis de las proposiciones genuinas, se ha de llegar a proposiciones elementales formadas por nombres simples (que denotarían objetos simples) dispuestos en conexión inmediata entre si (que representarían una posible configuración de objetos simples, $2.0231 ; 3.21)$, y que esto sería así "Aunque el mundo sea infinitamente complejo, de modo que cada hecho conste de infinitos estados de cosas, y cada estado de cosas, de infinitos objetos, aún entonces tendría que haber objetos y estados de cosas" (4.2211). W difiere a la aplicación de la lógica los detalles de tal tipo de análisis pero, en mi opinión, aunque esto resulte razonable respecto a la demanda de suministrar ejemplos de objetos simples, no puede excusar la ausencia de toda explicación respecto al modo de conexión entre los nombres simples, únicos componentes de las proposiciones elementales, pues el sentido mismo de las proposiciones está condicionado por las reglas de la sintaxis lógica y, en estas, el significado de los signos no ha de jugar papel alguno (3.33), sino que ellas mismas determinan qué combinaciones de signos son posibles (necesariamente posibles, 3.325; 6.124), y tal determina- 
ción ha de ser realizada a priori $(5.4731,5.552)$ y, por tanto, previamente a cualquier posible aplicación. Es por esto por lo que no resulta aceptable la excusa de W para no indicar el modo de concatenación de los nombres simples en la proposición elemental: "como no podemos aducir el número de nombres con significado diferente, tampoco podemos aducir la composición de la proposición elementaly (5.55), (sin cursiva en el original).

W estuvo lejos de haber logrado especificar con detalle un lenguaje lógicamente perfecto, por ejemplo, no son claras, como señaló Carnap (1934, p.50), las consecuencias de haber rechazado también la regla de substitución estándar de variables por constantes ${ }^{6}$; no obstante, sus indicaciones, respecto a éste, resultan suficientes para excluir del lenguaje ideal la expresión 'Sócrates es idéntico', y a las propias proposiciones del Tractatus, pues parece suficientemente claro que no son de la forma atribuida a las proposiciones elementales, ni resulta posible construirlas, empleando sólo conectivas (o cuantificadores), a partir de proposiciones de esa forma.

De este modo se ve por qué $W$ incluye en una misma categoría, la de las expresiones sinsentido, a 'Sócrates es idéntico' y a las pseudo-proposiciones del Tractatus: ambas resultan ser expresiones mal formadas por que no están construidas de acuerdo con las reglas del lenguaje ideal, la sintaxis lógica. De ahí que no formen parte de ese lenguaje y resulten sinsentidos consideradas desde el punto de vista de ese lenguaje.

Esto nos pone también en la pista de por qué W separa de esa categoría a tautologías y contradicciones: éstas son expresiones bien formadas de acuerdo con las reglas de la sintaxis lógica y, por tanto, al formar parte del lenguaje ideal, no cabe excluirlas de él como meros sinsentidos. Sin embargo, de su noción de sentido (4.2) se sigue que no pueden tener sentido (como lo tienen las proposiciones elementales y las moleculares contingentes) pues, sean cuales sean los estados de cosas que se dan en el mundo, su valor veritativo está determinado de antemano: verdadero, en el caso de las tautologías, y, falso, en el caso de las contradicciones. Por ello, ni unas ni otras logran describir ningún posible estado de cosas (que es lo que otorga sentido a una proposición); por ello carecen de sentido (Sinnlos), aunque no sean sinsentidos (Unsinnig).

6 Téngase en cuenta que si se mantiene la regla estándar de substitución de variables de individuo por constantes entonces la formalización ofrecida por W (en 5.5321) de 'Hay sólo un $x$ tal que Fx', a saber, ' $\exists x$ Fx $\cdot \neg \exists x y(F x \cdot F y)$ ', implica una contradicción. 
En resumen, W, en el Tractatus, es plenamente coherente en su uso de la noción de sentido: oraciones con sentido, proposiciones genuinas, son solamente aquellas que describen un posible estado de cosas, aquellas cuya verdad, o falsedad, depende de como sea de hecho el mundo; las proposiciones contingentes en suma. Todas aquellas oraciones que no cumplan esta condición, no tienen sentido (hat Keinen Sinn). A su vez, esta categoría se subdivide en otras dos categorías: de una parte estarían aquellas oraciones moleculares que resultan de modos legítimos de composición de proposiciones elementales mediante las reglas de la sintaxis lógica (tautologias y contradicciones (Sinnlos)); de la otra, estarían aquellas oraciones (Unsinnig) que no pueden ser construidas de este modo, y, por tanto, no formarían parte del lenguaje lógico ideal destinado a representar cómo es el mundo, (las propias pseudoproposiciones del Tractatus entre otras).

Ahora bien, por muy coherente que resulte el uso que hace $\mathrm{W}$ de los calificativos de 'carente de sentido' y de 'sinsentido' no cabe duda que continúa resultando extraño ver incluidos bajo ese rótulo común a oraciones como ' 1 es un número' o las propias oraciones del Tractatus y la extrañeza aumenta cuando reparamos en que expresiones claramente mal formadas como 'Sócrates es idéntico' forman parte de la misma categoría que las anteriores.

Pero sea cual sea el grado de extrañeza que produzcan tales conclusiones una cosa sí es clara: Hay una patente discrepancia entre el uso que hace $W$ de las expresiones 'carente de sentido' y 'sinsentido' y el modo en el que se usan estas expresiones en el lenguaje ordinario como advirtió Moore (1983, p. 274 ss.) en su momento. Ningún hablante competente del castellano calificaría de sinsentido una tautología como la expresada en la oración 'Lo que es indiscutible es que la chica está embarazada o no lo está, cuando concurren las circunstancias apropiadas no muy difíciles de imaginar. Tampoco se aplicaría tal calificativo a la contradicción contenida en la oración 'Es imposible que te haya pagado la deuda y que aún esté pendiente de saldar'. (Obsérvese que, si la contradicción fuera un puro sinsentido, entonces la oración resultante de anteponerle la expresión 'Es imposible que' también debería serlo.) Y, finalmente, tampoco creo que calificarían de sinsentidos a las oraciones que componen el Tractatus (;si no a todas al menos a muchas de ellas!).

Como he intentado mostrar, el origen de la discrepancia es claro: W, en el Tractatus, tiene en mente a la Lógica, concebida como sintaxis pura, como el paradigma de la racionalidad, como el molde que determina los límites del sentido: 
Todo lo que caiga fuera de él no es más que puro sinsentido pese a que pueda parecer otra cosa expresado en el lenguaje ordinario. Posteriormente, W llegará a considerar todo esto una mixtificación del lenguaje lógico que, fruto de una visión unilateral sobre el lenguaje, acaba ofreciendo una imagen empobrecida de la riqueza del lenguaje ordinario. Pero esto no se traducirá, como pudiera parecer, en una simple y llana aceptación de cualquier cosa que aparezca como expresión bien formada en el lenguaje ordinario. Como veremos persistirán las discrepancias sustantivas, aunque ahora ya no serán la consecuencia de haber considerado el lenguaje de la lógica, o cualquier otro lenguaje formal, como paradigma que determine los límites del sentido. Las discrepancias surgirán cuando al optar, implícitamente, por ciertos usos ordinarios frente a otros que resultan ser incongruentes con los elegidos, se acaba empleando reglas diferentes para operar con los mismos términos.

En definitiva, tanto si se considera afortunado el uso que hace W del término 'sinsentido', como si no, lo que hemos mostrado es que $W$ tiene razones filosbficas para apartarse de su modo de empleo en el lenguaje ordinario, y, por consiguiente, no puede mantenerse, consistentemente, que W se limite a seguir el uso ordinario de términos que juegan un papel central en su filosofia.

\section{III}

En la etapa madura de su obra, Wittgenstein parece oscilar entre dos concepciones de la filosofia: una a la que podriamos denominar 'descriptivista' y que ha sido resaltada por la mayoría de los comentadores hasta el punto de presentarla, en ocasiones, como la única concepción de W, o, al menos, del segundo W, y otra, que ha pasado más inadvertida, a la que podemos denominar 'constructivista' a falta de otro nombre mejor.

La concepción descriptivista es de sobra conocida y aparece paradigmáticamente reflejada en el tan citado párrafo 124 de las Investigaciones:

La filosofia no puede en modo alguno interferir en el uso efectivo del lenguaje; puede a la postre solamente describirlo.

Pues no puede tampoco fundamentarlo. Deja todo como está. (sin cursivas alli).

Esta concepción, tan tranquilizadora para algunos, resulta inadmisible para otros. El siguiente texto de M. Dummett resulta bastante representativo de la actitud de estos últimos: 
Usted conoce la famosa idea wittgensteiniana respecto a que la filosofia no puede alterar nada en absoluto, sólo puede describir, etc.

En particular, tenía la idea de que no se podía alterar la práctica lingüistica, y se vio envuelto en el absurdo de que la filosofía no podía alterar la práctica lingüística, incluso si se mostraba que esa práctica era inconsistente o llevaba contradicción. Se tienen que aceptar las contradicciones como un hecho. (Dummett en Pataut (1998, 119); sin cursivas allí).

No puede reprochársele a Dummett ni, en rigor, a nadie, que le atribuya a W tal concepción descriptivista, pues está fuera de toda duda que éste la reiteró permanentemente; el problema es más bien que la propia práctica filosófica de W no se atuvo en todo momento a esa concepción, sino que, como hemos visto antes y continuaremos apreciando a continuación, en algunos puntos importantes W se aparta del uso ordinario y llega a considerar sinsentidos oraciones que los hablantes corrientes nunca considerarían así. Por ello puedo estar de acuerdo con Dummett en que

No creo que hubiera ninguna justificación en su afirmación [de Wittgenstein] de que la filosoffa no puede interferir en nada.

Nuestra practica lingüística puede ser tan incorrecta como nuestra conducta puede ser incorrecta o irracional. La filosofia tiene el derecho de señalarlo. (op.cit., 121; sin cursiva alli)

sin dejar de reconocer, por esto, que W no siempre actuó de acuerdo con esa afirmación, y que, en concreto, y en contra de lo que Dummett le atribuye en la misma página, W admitió la posibilidad de cambiar las leyes lógicas, como creo haber mostrado en (1995).

Pero no se trata sólo de que W no se limitara, de hecho, a filosofar dentro del estrecho marco de la concepción descriptivista de la filosofía, sino que mantengo que existen textos suyos que justifican la atribución de una concepción más constructiva.

Efectivamente, la concepción constructivista ha pasado más desapercibida ${ }^{7}$ pero no por ello resulta menos importante para comprender el quehacer del

7 La única excepción que conozco (aunque no habla de constructivismo) es KENNY, quien reconociendo el papel crítico (o negativo) que W asigna a la filosofia (Invest., 118), no obstante 
propio W. Aparece ya claramente presupuesta en sus clases en Cambridge cuando retornó de nuevo al trabajo filoś́fico académico durante los primeros años de la década de los treinta. Así, cuando para aclarar la naturaleza de los problemas filosóficos, recurre al símil de la diferencia que existe entre ser capaz de orientarse y caminar a lo largo de un país, y ser capaz de elaborar un mapa de él (1979a, p.43). El mapa no se encuentra ahí a la espera de ser descubierto y descrito; el mapa hay que elaborarlo, construirlo: es el resultado de nuestra propia actividad y podemos equivocarnos al hacerlo.

En este símil el aspecto constructivo de la actividad se encuentra bastante limitado por el requisito de que el mapa ajuste, o represente adecuadamente, el país pretendido. Sin embargo, ese tipo de limitación se sobrepasa cuando lo que se hace no es meramente describir el lenguaje, sino inventar nuevos juegos de lenguaje como hicieron en su momento los químicos: "Pero no es historia natural inventar lenguajes propios, como he hecho, y establecer reglas para tales lenguajes, como hicieron, por ejemplo, los químicos del siglo XIX con el lenguaje de la Química.» (op. cit., p.97); y W tiene una justificación para ese modo de proceder: "Algunas veces establezco nuevas reglas porque las nuevas reglas son menos propicias a producir confusión o porque, quizás, no hemos pensado en mirar con esta luz al lenguaje que tenemos.»(op.cit.,p.97; sin cursivas alli.), y, al hacerlo así, podemos resaltar aspectos del lenguaje que antes podíamos haber pasado por alto; por ejemplo, la distinción entre explicar la conducta mediante razones o por causas.

Esta concepción constructivista no aparece solamente en los inicios de su nuevo modo de concebir el lenguaje, sino que también se encuentra presente en la que se ha considerado su fase madura, en las Investigaciones:

Interpretas la nueva concepción como el ver un nuevo objeto [la habitación visual]. Interpretas un movimiento gramatical que has hecho como un fenómeno cuasi-fisico que has observado. (Piensa, por ejemplo, en la pregunta: «¿Son los datos sensoriales la materia prima del universo?»)

sefiala que «uno puede encontrar en los escritos de WITTGENSTEIN evidencia de lo que, en principio, parece una perspectiva diferente y más positiva de la filosofia; una perspectiva de la filosofia como proporcionando un tipo especial de comprensión, una perspectiva muy general del mundo, una comprensión global" KENNY $(1984,42)$. Esa contraposición empleada por Kenny también la utiliza STRAWSON (1992, cap. 1), pero - siguiendo el proceder habitual_ sólo le adjudica a W la mitad de ella; la otra, la concepción positiva de la filosofia, sería la que él (STRAwSON) encuentra 'más atractiva' como tarea de la filosofia, en contraposición a WITTGENSTEIN. 
Pero mi expresion no es inobjetable: La de que bas becho un movimiento 'gramatical'. Ante todo has encontrado una nueva concepción. Es como si hubieras inventado una nueva forma de pintar; o también un nuevo metro, o un nuevo tipo de canto. (1988a, 401; sin cursivas alli).

En este sentido la reforma del lenguaje no está excluida (¿cómo podría excluirse tal cosa?, ¿qué sentido tendría la prohibición de reformarlo? ${ }^{8}$ ), aunque no sea tal reforma el propósito de W (op. cit.,132). El aspecto constructivo de la tarea filosófica aparece de nuevo implícito, pero claro, cuando nos dice "Queremos establecer un orden en nuestro conocimiento del uso del lenguaje» (op.cit., 132). Obsérvese que dice 'establecer' [herstellen], no 'describir' [beschreiben]; esto es, el orden no se descubre sino que se establece, se construye; y se construye con vistas a un fin: clarificar el uso del lenguaje, evitar malentendidos. Y es claro que al logro de ese fin no pueden establecérsele limitaciones teóricas de ningún tipo (¿qué podría constituir un ejemplo de tal tipo de limitación?). Así, el filósofo puede establecer distinciones allí donde resulten útiles para clarificar un problema; por ejemplo, la distinción entre actos ilocutivos y perlocutivos (Austin),o entre condicionales fácticos y contra-fácticos, o entre axiomas y definiciones,o entre enunciados descriptivos y prescriptivos (o usos de oraciones como descripciones y como prescripciones) o, finalmente,y por citar dos de las distinciones más queridas por W, la distinción entre explicar por razones o por causas, y la distinción entre enunciados descriptivos y enunciados gramaticales o reglas.

Pero no se trata solo de que el filósofo pueda establecer distinciones que ayuden a clarificar el uso ordinario de los términos, sino que mantengo que W se aparta explícitamente del uso ordinario de ciertos términos centrales para una adecuada comprensión del mundo. Hemos visto antes un caso de este tipo cuando atendíamos al uso del término 'sinsentido' en el Tractatus; ahora veremos otro a propósito de una noción que, en lo esencial, permanece constante a lo largo de su obra: la noción de verdad.

8 RUSSELl (1959,178ss.) no sólo consideró posible la reforma del lenguaje ordinario, sino indispensable, si se quiere obtener mayor precisión en lo que decimos. Si creía, como parece, que esta crítica alcanzaba al segundo WITTGENSTEIN, se equivocaba (cf.,Invest.,132); como se equivocó, mas claramente aún, en sus consideraciones generales (op.cit., pp. 160-1) sobre este. (Otra defensa, ya clásica, de la necesidad de reformar el lenguaje ordinario se encuentra en G. MaXwell y H. Feigl (1960, 193-200). La réplica de M. Thompson $(1960,201-5)$ a estos últimos no me parece que logre su objetivo, pues se apoya en un supuesto harto dudoso, a saber, la distinción entre creencia y lenguaje). 
IV

Uno de los puntos en los que aparece más claro que W se aparta del uso ordinario, se encuentra en su concepción de la noción de verdad.

Resulta bastante patente que en el lenguaje ordinario las siguientes oraciones tendrían pleno sentido, esto es, no serían tachadas como sinsentidos por los hablantes competentes del castellano, ¡excluidos los filósofos! (aunque no por incompetentes, sino por su inveterada y loable costumbre de discrepar):

(1) Es verdad que el aborto debe estar prohibido.

(2) Es verdad que el aborto está prohibido.

(3) Es verdad que el aborto no debe estar prohibido.

(4) Es verdad que el aborto no está prohibido?.

Sin embargo, desde el punto de vista de W, sólo (2) y (4) tendrían sentido, mientras que (1) y (3) serían, propiamente hablando, sinsentidos ${ }^{10}$ (aunque importantes desde un punto de vista filosófico). Desde luego no es posible citar ningún texto de $\mathrm{W}$ en el que éste mantenga explícitamente la opinión que le atribuyo (al menos que yo sepa), pero me parece claro que constituye una consecuencia inevitable de su concepción de la verdad, tanto en el Tractatus, como en el resto de su obra. No obstante, parece claro que esa posición que le atribuyo se encuentra, al menos, implícita, cuando dice:

Todo lo que describo está dentro del mundo. Una proposición ética nunca aparece en la completa descripción del mundo, ni aún cuando describo un asesinato.

Lo que es ético no es un estado de cosas. (1979 b, 92).

9 Elijo esa formulación porque me parece más idiomática que la formulación equivalente: "El aborto no está prohibido" es verdad.

10 A lo largo de lo que se ha dado en considerar la segunda etapa en el pensamiento de W,éste se desentiende de la distinción, que mantenía en el Tractatus, entre expresiones carentes de sentido (Sinnlos) y expresiones sinsentido (Unsinn), usando ahora, indistintamente, ambas expresiones para calificar a aquellas combinaciones de palabras que, por unas u otras razones, no forman parte del lenguaje, pese a que pueda parecer lo contrario; por ejemplo,'Hay objetos físicos' (1988b, 35). [Mas detalles sobre esto se encuentran en GLOCK (1996, 258-264)]. 
(Conviene advertir, sin embargo, que, en el contexto en el que aparece esa cita, no hay nada que sugiera que $\mathrm{W}$ pensara que el aborto constituía un asesinato).

Una de las constantes del pensamiento de W, a lo largo de toda su obra, consiste en la idea de que el par verdadero-falso solo es aplicable a proposiciones o enunciados declarativos [Sätze]. Mas ¿qué es una proposición?. Según W, la forma general de una proposición es: 'Las cosas están así y asá' (Tract.,4.5; Invest., 136). Es decir, las proposiciones dicen, o describen, como son, o están, las cosas en el mundo. Por esto, podemos decir que "una proposición es todo lo que puede ser verdadero o falso. Pues en vez de 'Las cosas están ...' podría también haber dicho: 'Esto y aquello es verdadero'. (Pero también: 'Esto y aquello es falso'.)» (op. cit., 136)

Así, las proposiciones aparecen definidas como aquello de lo que predicamos (podemos predicar) los términos 'verdadero' y 'falso'. Pero es claro que no de toda la oración bien formada podemos predicar, con sentido, tales términos; por ejemplo, no tendría sentido predicarlo de la oración interrogativa ‘Cuantos años tienes?', ni de la imperativa 'Cierra la puerta' (aunque, como siempre, haya filósofos que discrepen de lo que acabo de indicar ${ }^{11}$ ); es decir, aunque tales oraciones tienen pleno sentido, carecerían de él las expresiones "Cierra la puerta' es falso' (en oposición a "La puerta está cerrada' es falso'), y “¿Cuantos años tienes?" es verdadero' (en oposición a "Tienes seis ańos' es verdadero'), que resultan de añadir 'es falso' y 'es verdadero', respectivamente, a las oraciones anteriores.

Parece que W estaba teniendo en cuenta ejemplos como los anteriores cuando escribió:

Se podría enseñar a un niño a distinguir proposiciones de otras expresiones diciéndole: 'Pregúntate si puedes decir 'es verdadero' después de ello. $\mathrm{Si}$ estas palabras ajustan, entonces es una proposición'. (Y del mismo modo se hubiera podido decir: Pregúntate si puedes colocar las palabras 'Las cosas están ast:' delante de ello. (1988a, 137).

11 DAVIDSON constituye un caso paradigmático de esto. En mi opinión extender el uso de 'verdad' como propone DAviDSON (1984, p. 31), de modo que resultaría aplicable a oraciones evaluativas como 'Bardot es buena' o imperativas como 'Cierra la puerta' sólo contribuye a perpetuar la confusión trasladándola ahora al metalenguaje (como parece reconocer el propio Davidson). Por su parte, BAKER y HACKER $(1984,48)$ califican directamente de nonsense tal tipo de intentos. 
Ahora bien, como indicábamos antes, constituye un hecho lingüístico acerca del uso del lenguaje, que los hablantes competentes de éste aceptarian que tanto (1), como (3), son oraciones con sentido; y no serían pocos los que considerarían que sus creencias estarían adecuadamente representadas por (1), mientras que otros muchos aceptarían (3) como resumen de su posición respecto al importante tema del aborto. Mas aún, creo que tanto unos como otros considerarían que habría una inconsistencia, o incompatibilidad, si alguien dijera que admite simultáneamente (1) y (3).

Sin embargo, desde un punto de vista wittgensteiniano habría que decir que tanto (1) como (3) carecen de sentido. Asl que el problema inmediato es: ¿Cómo justificar (si es posible tal cosa), desde un punto de vista wittgensteiniano, esa conclusión que choca abiertamente con el uso ordinario del lenguaje?.

La clave de la justificación se encuentra en la noción de proposición que $W$ maneja. Efectivamente, una proposición es aquello que dice como son, o están, las cosas; así que si las cosas son, o están, como dice la proposición que están, esta será verdadera, y si las cosas no son, o no están, como ella dice entonces será falsa. Ahora bien, como véamos antes, no toda oración con sentido realiza la función de indicar como son, o están, las cosas, sino que algunas lo que indican es, no como son, sino como deberian ser, tanto si son así como si no; por ejemplo, son de este tipo las oraciones:

(1') El aborto debe estar prohibido.

(3') El aborto no debe estar prohibido.

Que tanto (1') como (3') no realizan (no se usan para realizar) la función de indicar cómo están las cosas se muestra atendiendo al hecho de que ninguna de ellas sería inconsistente con cualquiera de las dos oraciones:

(2') El aborto está prohibido.

(4') El aborto no está prohibido,

que sí pretenden indicar, o describir, cómo están, de hecho, las cosas respecto a la legislación sobre el aborto en determinada sociedad en un momento dado.

Tanto (2') como (4') se usan para describir como están las cosas, y, por ello, tiene pleno sentido calificarlas de verdaderas o de falsas; pero ( $\left.1^{\prime}\right)$ y ( $\left.3^{\prime}\right)$ no describen nada, sino que se usan para prescribir, o recomendar, como tendrían que estar las cosas, independientemente de que estén en el caso representado por 
(2') o en el caso representado por (4'). Es decir, tanto si creemos que las cosas son como dice ( $\left.2^{\prime}\right)$ que son o como dice (4'), tiene pleno sentido, respecto a esta situación, aceptar ( $\left.1^{\prime}\right)$ o aceptar ( $\left.3^{\prime}\right)$; esto es, no hay ninguna inconsistencia entre creer (2') y aceptar (1'), o (3'), de una parte, o bien creer (4') y acep$\operatorname{tar}\left(1^{\prime}\right)$, o (3'), de la otra. El punto crucial es, pues, que siempre se puede decir: 'Así es como están las cosas y así es como deben estar', y también 'Así es como están las cosas, pero así no deben estar', sin que resulte redundante en el primer caso, ni contradictorio en el segundo. Así que, si admitimos que la situación respecto a la legislación del aborto sólo puede estar en uno de los dos casos, o permitida o prohibida, (¿y como podría negarse esto?) y comprobamos que tanto (1') como (3') son consistentes (por separado) con cualquiera de las dos situaciones posibles, entonces es claro que ni (1') ni (3') son el tipo de oración del que tenga propiamente sentido decir que son verdaderas o falsas; pues estos términos sólo son aplicables a las oraciones que pretenden indicar, o describir, cómo son las cosas ${ }^{12}$.

Ese es el modo en el que creo que puede justificarse, desde una perspectiva wittgensteiniana, la tesis de que los términos 'verdadero' y 'falso' no son aplicables a oraciones del tipo ( $\left.1^{\prime}\right)$ y ( $3^{\prime}$ ), y que justificaría, por consiguiente, que se consideren como sinsentidos las oraciones (1) y (3).

Acabo de señalar cómo se justificarían desde una perspectiva wittgensteiniana, pero ahora la cuestión capital e ineludible es: ¿Cómo justificar (si es posible) tal perspectiva?, esto es, ¿cómo justificar ese tipo de análisis cuya conclusión choca frontalmente con lo que dicen los hablantes en el lenguaje ordinario?.

En mi opinión, la respuesta correcta a esta pregunta (por paradojica que pueda resultar a primera vista) es que el análisis de W del uso de los términos 'verdadero' y 'falso' representa adecuadamente el núcleo esencial de su uso en el lenguaje ordinario.

12 Podría objetarse aquí que W mantiene que (V) Es verdad que $\mathrm{p}=\mathrm{p}, \mathrm{y}$, así, si la oración que ocupa la posición de 'p' en (V) tiene sentido, también lo tendría, para W, la oración resultante de anteponerle 'Es verdad que'. Pero el punto que no se tiene en cuenta en esta objeción es que esa ecuación no la mantiene W para toda oración. Obviamente (V) carece de sentido si 'p' es sustituida por oraciones interrogativas como '¿Cuando te licenciaste?' o imperativas como 'Pásame la sal' y, como argumento en el texto, creo que W tampoco admitiría la aplicación de (V) al caso de oraciones que no sean descriptivas de como están las cosas en el mundo; en concreto, argumento que no lo aplicaría a oraciones valorativas como 'El asesinato es malo' (1979b, 92) o prescriptivas de la forma 'Debes....'. 
Esto es, lo que estoy afirmando es que, en el lenguaje ordinario, aplicamos los términos 'verdadero' y 'falso' ante todo y sobre todo a las oraciones que describen cómo son, o están, las cosas. A oraciones como 'Lleva calcetines verdes de lana', 'Le han robado el coche', 'Está deprimida', o a oraciones como (2') y (4'). A este respecto no hay divergencia entre lo que dice $W$ y lo que decimos en el lenguaje ordinario. Aquí el 'ajuste' es completo. Sin embargo, en el lenguaje ordinario se produce una extensión en el uso de los términos 'verdadero' y 'falso', y se aplica también, como venimos subrayando, a oraciones que no funcionan como descripciones de cómo son las cosas; testigos de esto son (1) y (3). Lo que ocurre es que en esa extensión hemos perdido el fundamento sobre el que descansaba la atribución de verdadero y falso a las oraciones. En mi opinión, este tipo de extensión resulta análogo al que, en otro contexto, W denuncia con claridad:

Es como si yo dijese: "Tu sabes por cierto lo que quiere decir 'Son aquí las 5 en punto'; luego sabes también lo que quiere decir que son las 5 en punto en el Sol. Quiere decir justamente que allí es la misma hora que aquí cuando aquí son las 5 en punto". (1988 a, 350).

Aplicábamos 'verdadero' o 'falso' a (2') y a (4') porque tanto uno como otro enunciado funcionaban como descripciones de como son las cosas. Pero ni (1'), ni (3'), funcionaban de ese modo según hemos visto; así que si ahora admitimos que también tiene sentido aplicar tales términos a estas últimas oraciones, entonces nuestro fundamento para hacerlo así ha de ser diferente del que tenemos para aplicárselo a (2') y (4'). Y esto es tanto como decir que no estamos empleando 'verdadero' y 'falso' con el mismo significado cuando lo aplicamos a (2') y (4') de una parte y a (1') y (3') de la otra. Cuando lo aplicamos a (2') y (4') estamos indicando que así es como, efectivamente, son las cosas; cuando lo aplicamos a (1') y (3') ya no estaremos diciendo esto, sino otra cosa pendiente aún de determinar ${ }^{13}$. Pero esto es tanto como reconocer que aunque tengamos una única palabra, 'verdadero' (o 'falso') tendrá significados diferen-

13 Un excelente análisis sobre el significado de 'verdadero' y 'falso' cuando se aplica a enunciados de deber o, en general, a enunciados morales, se encuentra en R.M. HARE [1997, especialmente en pp. 48-60] Por otra parte, JOHNSTON (1989, pp. 142-3 y nota 3. p. 233) subraya, acertadamente, que, desde un punto de vista wittgensteiniano, sería una patente confusión entender 'verdadero' con el mismo significado dicho de un enunciado empírico y dicho de un juicio moral. 
tes cuando se aplique a (2') o (4') y cuando se aplique a (1') o a (3'), y, en este caso, como decía W a propósito de una situación similar, podemos cambiar la palabra también (1969, p.184).

Ahora bien, en el uso ordinario, hemos visto como la misma palabra 'verdadero' (o 'falso) se usa adjuntándola a oraciones que funcionan de modo radicalmente diferente, a (2') que se usa para describir, y a (1') que se usa para prescribir o recomendar. $Y$, en principio, no habría mayor problema en esto que en reconocer que estaríamos en este caso ante una palabra ambigua (como tantas otras en el lenguaje ordinario; 'banco' como ejemplo paradigmático); pero, por esto mismo, sería una confusión ${ }^{14}$ creer que la estamos empleando básicamente con el mismo significado en uno y otro caso según hemos visto. Wittgenstein indica con claridad una situación de este tipo:

Los casos en los que de un modo particular deseamos decir que alguien ha sido confundido por una forma de expresion son aquellos en los que diríamos: "no hablaría como lo hace si se diese cuenta de esta diferencia en la gramática de tal y tal palabra, o si se diese cuenta de esta otra posibilidad de expresión", etc. Así, podemos decir de varios de los matemáticos que filosofan que es evidente que no se dan cuenta de la diferencia existente entre los varios usos diferentes de la palabra "prueba»; y no ven con la suficiente claridad la diferencia entre los usos de la palabra "clase" cuando hablan de clases de números y clases de pruebas, como si la palabra "clase» significase aquí lo mismo que en el contexto "clases de manzanas". O podemos decir que no se dan cuenta de los diferentes significados de la palabra "descubrimiento" cuando en un caso hablamos del descubrimiento de la construcción del pentágono y en otro del descubrimiento del Polo Sur. (1968, pp. 57-58; sin cursivas alli, salvo la penúltima.)

Pues bien, toda mi tesis aquí es que en el uso de 'verdadero', en (1) y (3), subyace una confusión análoga a la que $\mathrm{W}$ acaba de señalar a propósito de los usos de las palabras 'prueba', 'clase' y 'descubrimiento'; y que la diferencia entre que

14 Creo que este tipo de confusión está en la base de una confusión más general y cuya importancia para la vida de cualquier persona resulta difícil de exagerar, a saber, la que surge al no distinguir entre estas tres cuestiones: cómo son las cosas, cómo deberían ser, y cómo desearíamos que fueran. Que habitualmente no coincidan las respuestas a ellas es fuente de infelicidad, y, que no se tenga conciencia de su diferencia, origen de evitables conflictos intra e ínter personales. 
sea un matemático al 'filosofar', o un hablante ordinario al hablar, no es relevante respecto a su posible confusión, o èes que hay algo que tiene el segundo, pero de lo que carece el primero, que lo salvaguarda de toda posible confusión? ${ }^{15}$

Efectivamente, tanto el que asiente a (1) como el que asiente a (3) negaría que esté entendiendo 'verdadero', respecto a su uso en (2) y (4), con tanta diferencia de significado como lo estaría la palabra 'banco' dicha del transportable banco de madera en el que nos sentamos y dicha de una institución de crédito. Pues, si fueran conscientes de la diferencia de significado de 'verdad' cuando se adjunta a (2'), y cuando se adjunta a (1'), entonces no resultarían comprensibles las acaloradas discusiones entre los que aceptan (1) y los que aceptan (3) tratando de sacar del error a su adversario, como si este no fuera capaz de percibir la realidad tal cual es; achacable, acaso, a que no le funciona bien el mecanismo de percepción moral a causa, quizás, de algún defecto orgánico de difícil corrección o, más piadosamente, a causa de alguna deficiencia en su aprendizaje que podría ser corregida por las buenas (o, por las malas, si ello se juzgara necesario para que finalmente vea la verdad - de (1') en el caso más frecuente-.)

Si a esto se objetara que el 'calor' de la discusión no implica, como parece presuponerse en la argumentación precedente, que 'verdad' se tome con el mismo significado en (1) que en (2) por ejemplo, siempre cabría alegar, prosiguiendo con la metáfora, que, aunque ciertamente hay muchas y variadas formas de calentarse, la que hemos indicado no es de las menos frecuentes entre los que defienden (1) y los que defienden (3).

Pero, sea esto como fuere, el punto importante es que W, por buenas razones según he tratado de exponer, se aparta del uso ordinario del término 'verdad' cuando, desde su perspectiva, se dictamina que ni (1) ni (3) tienen propiamente sentido. Esto no quiere decir, claro está, que $W$ se separe completamente de los usos ordinarios de 'verdad', entre otras cosas porque, si así fuera, entonces no habría ninguna razón para decir que hay discrepancias entre lo que

15 Incluso HACKER, que no se caracteriza precisamente por subrayar las confusiones latentes en el lenguaje ordinario, viene a reconocer que este no se encuentra libre de ellas: "La fuente primaria de superstición y confusión filosofica es el lenguaje mismo. Así tenemos que luchar contra el embrujo de nuestro entendimiento por medio del lenguaje [Invest., 109], contra las ilusiones gramaticales, y contra la mala interpretación de nuestras formas de lenguaje. Esto se aplica al lenguaje técnico de los matemáticos, flisicos y psicólogos, no menos que al lenguaje ordinario". HACKER (1996, 115;sin cursivas alli). 
él dice y lo que se dice en el lenguaje ordinario; estariamos, en este hipotético caso, ante dos usos de una misma palabra tan desconectados entre si como lo están los usos de 'banco' aplicado a instituciones de crédito y a agrupaciones de peces; pero, como hemos visto, hay discrepancias respecto a la extensión del empleo de 'verdadero' sin alterar el significado que parece tener en los casos en los que hay coincidencia entre su uso en el lenguaje ordinario y su uso siguiendo el análisis wittgensteiniano.

Precisamente porque hay esa coincidencia, aunque parcial, entre lo que $\mathrm{W}$ mantiene y lo que se dice en el lenguaje ordinario, y no hay ninguna referencia explícita por parte de $\mathrm{W}$ a su discrepancia con lo que se dice en aquél, es por lo que se ha propagado la infundada leyenda de que $\mathrm{W}$ no es más que un filosofo del lenguaje ordinario, que preso del dogma de que 'el lenguaje ordinario está bien como está trata a éste como cosa sacrosanta (en análoga actitud a la que otros adoptan ante la Iglesia, la Patria, la Raza, el Partido o, inclusive, la Ciencia); actitud esta última a todas luces incompatible con una auténtica actitud filosófica; pero ésta, nadie podría negársela a W con fundamento ${ }^{16}$.

Mi objetivo ${ }^{17}$, en este artículo, ha sido sobre todo mostrar lo equivocado de esa leyenda, aunque para ello haya resultado preciso mostrar que no se puede mantener, consistentemente, el análisis wittgensteiniano de la noción de verdad y el mencionado dogma, que $\mathrm{W}$ incumplio ${ }^{18}$ reiteradamente a lo largo de toda su obra, y no sólo a propósito de los casos examinados aquí. Así, por

16 Otra razón de que haya pasado desapercibida la oposición entre lo que W mantiene y lo que se admite como teniendo sentido en el lenguaje ordinario procede de que, como KRIPKE (1982, p.64ss.) ha señalado en otro contexto, W parece efectuar una maniobra análoga a la de BERKELEY, a saber, la de considerar el conflicto como aparente y fruto de una mala interpretación de lo que realmente se dice en el propio lenguaje ordinario.

17 Objetivo que resulta bastante modesto si lo comparamos con el de KENNY: «Intento mostrar que él [W de las Invest.] llegó a desfigurar el Tractatus respecto a la naturaleza de los nombres, la naturaleza de los objetos, la naturaleza de los hechos y respecto a la naturaleza de las proposiciones; con el efeto de que sobreestimó la distancia que separaba la teoria pictórica del significado de la teoría de las Investigaciones Filosofficas. Lo que Wittgenstein atacó en su trabajo posterior, mantengo, es, algunas veces, no el Tractatus real, sino un fantasma de éstem. Kenny $(1984, \mathrm{ix}-\mathbf{x})$.

18 Ayer también aprecia un desajuste, aunque de otro ripo, entre lo que $\mathrm{W}$ declara y lo que efectivamente hace: «Su repetida preferencia por la descripción antes que por la explicación, y la abstención de teorías que afirmaba practicar y recomendaba a sus lectores, no fueron rasgos caracteristicos de su proceder real en ninguna etapa de su desarrollo, ni siquiera en las Investigacionesn. Ayer (1986, 171; sin cursivas alli, excepto la última) 
ejemplo, W vuelve a separarse del uso ordinario, aunque por sólidas razones, como intenté mostrar en (1994) y (1995), cuando llega a la conclusión de que los habitualmente considerados enunciados matemáticos no son propiamente hablando enunciados declarativos, o proposiciones, susceptibles de verdad o falsedad, sino que, en rigor, expresan reglas de naturaleza convencional para el manejo de los signos matemáticos. Análogamente, W se separa de lo que decimos corrientemente cuando tacha de sinsentidos, o de cosas que no pueden decirse, a las oraciones: 'Hay infinitos números primos' (1964, p.330); 'Rojo es un color', 'Un círculo es una forma', 'Esta varilla tiene longitud' (1969, pp.61 y 129); 'El metro patrón de París mide un metro de longitud', 'Sé que tengo dolor' (1988a, 50, 246); 'Hay objetos físicos' y 'Es posible que esté soñando' (1988b, 35, 383). Sin embargo, en ninguno de estos casos, al igual que en los casos que hemos examinado con detenimiento anteriormente, el modo de proceder de $\mathrm{W}$ es arbitrario o infundado. En todos los casos mencionados dispone (en contra de lo que probablemente él mismo diría) de buenas razones filosofficas, para proceder como lo hace. Solo he intentado justificar esto último a propósito de un par de ellos; razones de espacio me han impedido ampliarlo a los demás casos; quede esto para otra ocasión ${ }^{19}$.

\section{Bibliografía}

ACERO, J.J. (1990): «Significado y necesidad en el Tractatus», $\Delta \alpha \mu \omega \omega v, 2,5-41$.

ALEMAN, A. (1985): Teoria de las categorias en la filosofia analitica, Madrid, Tecnos, 2." ed. 1996.

- (1994): «La noción de convención en Wittgenstein», Revista de Filosofia, VII, 369381.

- (1995): “Wittgenstein: Lógica, matemáticas y convención», Revista de Filosofia, VIII, 57-75.

AYER, A.J. (1986): Wittgenstein, Editorial Crítica. (Trad. de J. SEMPERE del original Wittgenstein, Londres, George Weindenfeld and Nicolson Ltd, 1985).

ARISTOTELES (1983): Categorias, Valencia, Teorema. (Edición griego-español con trad., introd. y notas de L.M. VALDÉS).

19 Agradezco a José Hierro sus atinadas sugerencias que han permitido mejorar el texto. 
BAKER, G.P. y HACKER, P.M.S. (1984): Language, Sense and Nonsense, Oxford, Basil Blackwell.

- 1992 (1985) Wittgenstein, Rules, Grammar and Necessity, Oxford, Basil Blackwell.

Carnar, R. (1934): The Logical Syntax of Language, Londres, Kegan Paul.

Davidson, D. (1984): Inquires into Truth and Interpretation, Oxford, Clarendon Press.

GARCIADIEgo, A. (1992): Bertrand Russell y los origenes de las 'paradojas' de la teoria de conjuntos, Madrid, Alianza Editorial.

Glock, H-J. (1996): A Wittgenstein Dictionary, Cambridge, Basil Blackwell.

HaCKER, P.M.S. (1996): Wittgenstein's Place in Twentieth-Century Analitic Philosophy, Oxford, Basil Blackwell.

HalletT, G. (1977): A Companion to Wittgenstein's "Philosophical Investigations", Londres, Cornell University Press.

HARE, R.M. (1997): Sorting Out Ethics, Oxford, Clarendon Press.

HierRo, J. (1992): "La idea de mostrar en el Tractatus de Wittgenstein», Theoria, VII, 859-874.

IsHIGURO, H. (1981): "Wittgenstein and the Theory of Types", pp. 43-59; (incluido en I. Block (ed.) Perspectives on the Philosophy of Wittgenstein, Oxford, Basil Blackwell, 1981).

JoHnston, P. (1989): Wittgenstein and Moral Philosophy, Londres, Routledge.

KENNY, A. (1984): The Legacy of Wittgenstein, Oxford, Basil Blackwell.

KRIPKE, S. (1982): Wittgenstein on Rules and Private Language, Cambridge, Harvard University Press.

MAXWELl, G. y FeIGL, H. (1960): "Why Ordinary Language Needs Reforming»; (incluido en RORTY, R. (ed.) 1967; por donde cito).

MOORE, G.E. (1983): Defensa del sentido común y otros ensayos, Barcelona, Ediciones Orbis. (Trad. de C. Solís del original : Philosophical Papers, Londres, G. Allen and Unwind, 1959).

PATAUT, F. (1998): «Una perspectiva antirrealista sobre el lenguaje, el pensamiento, la lógica y la historia de la filosofla analítica: entrevista con Michael Dummett", Teorema, XVII/2, 115-44. (Trad. de P. GarClA del original publicado en Philosophical Investigations, 19/1, 1-33, 1996).

RoRTY, R. (ed.) (1967): The Linguistic Turn, The University of Chicago Press.

RusSELL, B. (1959) (1993): My Philosophical Development, Londres, Routledge (Trad. J. Novella: La evolución de mi pensamiento filosófico, Madrid, Alianza, 1976) 
STRaWsON, P.F. (1992): Analysis and Metaphysics, Oxford University Press.

ThOMPson, M. (1960): «When Is Ordinary Language Reformed?»; (incluido en Rorty, R. (ed.) 1967; por donde cito).

WITTGENSTEIN, L. (1921): Tractatus logico-philosophicus. (Edición alemán-español; trad. e introd. de J. MuÑoz e I. REguerA. Barcelona, Altaya, 1994; por donde cito).

- (1964): Philosophische Bemerkungen, Londres, Blackwell.

- (1968): Los cuadernos azúl y marrón, Madrid, Tecnos (Trad. de F. GARCí del original: The Blue and Brown Books, Oxford, Basil Blackwell, 1958).

- (1969): Philosophiche Grammatik, Londres, Blackwell.

- (1979a): Wittgenstein's Lectures, Cambridge 1932-35, Oxford, Blackwell. (Editado por AMBROSE de las notas tomadas por A. AMBROSE y M. MACDONALD).

- (1979b): Ludwig Wittgenstein and the Vienna Circle, Oxford, Blackwell. (Notas tomadas por F. WAISMANN y editadas por B. MCGUINNESS).

- (1988a): Investigaciones filosofficas, Barcelona, Crítica. (Edición alemán-español; trad. A. Garcla y U. Moulines).

- (1988b): Sobre la certeza, Barcelona, Gedisa (Edición alemán-español; trad. J.LL. PRADES Y U. RAGA). 